\title{
Avaliação da capacidade de fixação de fósforo pelo solo $\left(^{*}\right)$
}

\author{
R. A. CATANI e D. PELLEGRINO
}

Escola Superior de Agricultura "Luiz de Queiroz»

* Recebido para publicação em 24 de abril de 1960. 


\section{1 - INTRODUÇÃO}

Fixação é um têrmo genérico, empregado para traduzir a transformação de formas solúveis do fósforo em outras formas menos solúveis. Qualquer fenômeno que determine um decréscimo na concentração do ion ortofosfato de uma solução, em contato com o solo, é responsável pela "fixação". Vê-se, portanto, que as causas que concorrem para a fixação do fósforo, podem ser inúmeras.

Diversos autores fizeram uma revisão da literatura sôbre as causas e conceitos concernentes à fixação do fósforo pelo solo, (DEAN, 1949; KURTZ, 1953; OLSEN, 1953; HEMWALL, 1957) e outros. Entretanto, em resumo, pode-se concluir que as opiniões são concordes que para os solos ácidos, os compostos de ferro e alumínio são os maiores responsáveis pela fixação, ao passo que, para os solos alcalinos, são os compostos de cálcio.

Para os solos do Estado de S. Paulo, onde os sistemas de óxidos hidratados de ferro e alumínio ocorrem em proporção relativamente elevada, tem-se verificado com fósforo radioativo $P_{3}$, e fósforo comum $P_{31}$. que o ín fosfato é fixado mesmo em solução de $\mathrm{H}_{2} \mathrm{SO}_{1} 0,05$ normal (CATANI \& PELEGRINO, 1957).

A quantidade de fósforo fixada por um solo, depende de uma série de fatôres, como: concentração de fosfato da solução, $r H$ da solução, tempo de reação, temperatura, relação entre o nêso da amostra de solo estudado e volume da solução, e características ffsico-químicas do próprio solo.

Todos os métodos de avaliação da capacidade de fixação do fósforo pelo solo são convencionais e fornecem resultados variáveis, de acôrdo com as condições do trabalho.

A finalidade do presente trabalho é apresentar os dados obtidos relativos à fixação do fósforo para uma série de 15 amostras de terras ricas em óxidos hidratados de ferro e alumínio (terra roxa e roxa misturada). empregando também um método convencional.

\section{2 - MATERIAL E MÉTODOS}

O material estudado constituiu-se de 15 amostras de terra que, aparentemente, podeniam ser classificadas como roxas e roxas misturadas. As caracteristicas mais importantes das terras achamse resumidas nos quadros 1 e 2 . 
QUADRO 1 - Análise granulométrica das amostras estudadas

\begin{tabular}{c|c|c|c|c}
\hline \multirow{2}{*}{ N. } & $\begin{array}{c}\% \\
\text { areia }\end{array}$ & $\begin{array}{c}\% \\
\text { grossa }\end{array}$ & $\begin{array}{c}\% \\
\text { areia fina }\end{array}$ & $\begin{array}{c}\% \\
\text { argila }\end{array}$ \\
\hline 1 & 6,6 & 20,7 & 27,7 & 45,0 \\
2 & 17,3 & 24,2 & 21,4 & 37,1 \\
3 & 9,6 & 25,0 & 22,0 & 43,4 \\
4 & 10,5 & 25,1 & 28,0 & 38,4 \\
5 & 5,5 & 30,9 & 40,4 & 23,2 \\
6 & 23,4 & 15,6 & 17,4 & 43,6 \\
7 & 21,3 & 15,4 & 21,8 & 41,5 \\
8 & 23,3 & 21,4 & 20,3 & 35,0 \\
9 & 20,2 & 7,0 & 22,8 & 49,0 \\
10 & 14,1 & 32,3 & 26,0 & 27,6 \\
11 & 3,5 & 31,7 & 16,0 & 48,8 \\
12 & 4,0 & 17,3 & 12,6 & 66,1 \\
13 & 49,6 & 30,7 & 2,7 & 17,0 \\
14 & 8,4 & 24,4 & 12,2 & 54,9 \\
15 & 8,4 & 24,9 & 16,4 & 50,2 \\
\hline
\end{tabular}

$\mathrm{Na} 1^{*}$ coluna do quadro 2 estão os números das amostras da terra. $\mathrm{Na} 2^{\text {s }}$ coluna figuram os valores do $\mathrm{pH}$, obtidos em suspensão de $10 \mathrm{~g}$ de solo e $25 \mathrm{ml}$ de água destilada. Na $3 .^{\mathrm{a}}$ coluna e na $4^{\mathrm{a}}$. são apresentados os teores totais de carbono e nitrogênio em pêso. Nas $5^{\mathrm{h}}$ e $6^{\mathrm{a}}$ colunas, os teores em e.mg de $\mathrm{PO}_{4}^{-3}$ por $100 \mathrm{~g}$ de solo, extraídos, respectivamente, com $100 \mathrm{ml}$ de solução de $\mathrm{H}_{2} \mathrm{SO}_{4} 005$ normal e $100 \mathrm{ml}$ de solução 0,025 normal em $\mathrm{NH}_{4} \mathrm{~F}$ e 0,05 normal em $\mathrm{H}_{2} \mathrm{SO}_{4}$. Os dados obtidos com a solução de $\mathrm{H}_{2} \mathrm{SO}_{4}$ estão assinalados por $\left({ }^{1}\right)$ na $5^{\text {a }}$ coluna e os obtidos com solução $0,025 \mathrm{~N} \mathrm{em} \mathrm{NH}_{4} \mathrm{~F}$ e $0,05 \mathrm{~N} \mathrm{em} \mathrm{H}_{2} \mathrm{SO}_{4}$, estão identificados por $\left(^{2}\right)$. Na $7^{a}, 8^{a}, 9^{a}$ e $10^{a}$ colunas, os teores trocáveis em e.mg por $100 \mathrm{~g}$ de solo de potássio, cálcio, magnésio e hidrogênio, respectivamente. $\mathrm{Na} 11^{\text {a }}$ coluna, a soma $s$ de bases, $\mathrm{K}^{+}, \mathrm{Ca}^{+2} \mathrm{e} \mathrm{Mg}^{+2}$, em e.mg por $100 \mathrm{~g}$ de solo. $\mathrm{Na} 12^{\text {a }}$ coluna. a capacidade de troca de cátions $t$, em e.mg por $100 \mathrm{~g}$ de solo. Finalmente, na $13^{\text {n }}$ coluna, o indice de saturação $i$, calculado mediante s/t.100.

$O$ método empregado para avaliar a capacidade de fixação do fósforo do solo é convencional, conforme já foi esclarecido. Resultou do estudo de vários fatôres, como concentração de fosfato da solução, $\mathrm{pH}$, relação entre o pêso da amostra e volume de solução, tempo de agitação, tempo de contato com o solo, etc.

A técnica empregada foi a seguinte:

$4 \mathrm{~g}$ de terra foram agitados em balão de Erlenmeyer de $300 \mathrm{ml}$, durante 15 minutos a 30-40 rpm em aparêlho tipo Wag- 


\begin{tabular}{|c|c|}
\hline $\begin{array}{r}8 \\
-\quad n 1+\end{array}$ & $\begin{array}{l}0 \\
\infty \\
0 \\
0\end{array}$ \\
\hline$+\stackrel{\infty}{E}_{i}^{\infty}$ & 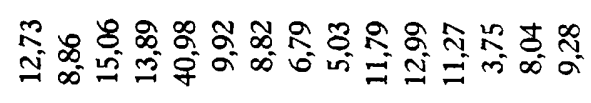 \\
\hline is $\stackrel{\infty}{E}_{i}^{0}$ & 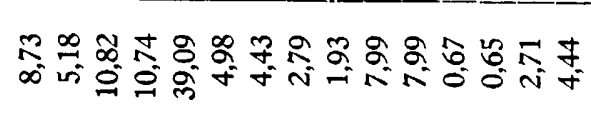 \\
\hline 声 $\underset{0}{\stackrel{Q}{E}} 20$ & 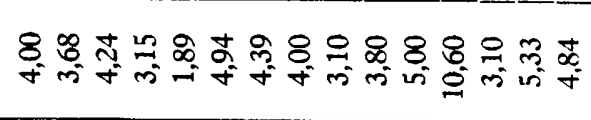 \\
\hline 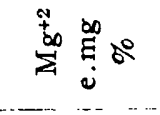 & 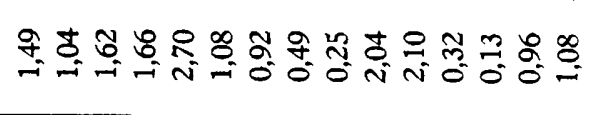 \\
\hline نึ & $\begin{array}{l}\approx \\
0\end{array}$ \\
\hline 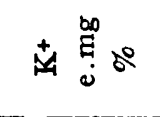 & 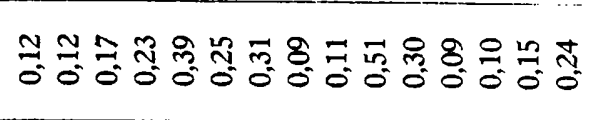 \\
\hline (ิ) & 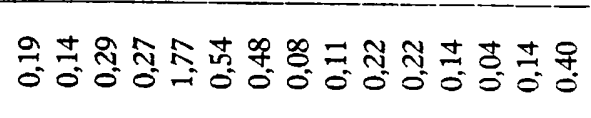 \\
\hline હ & 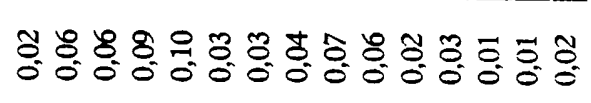 \\
\hline z 20 & 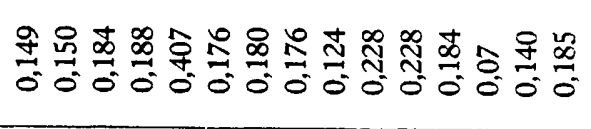 \\
\hline$u 80$ & 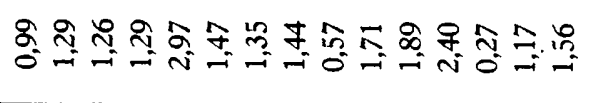 \\
\hline 寀 & 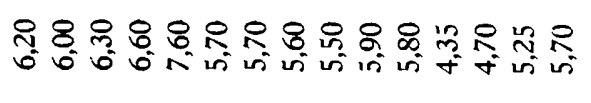 \\
\hline$\dot{z}$ & $-N m+n b N \infty a=ㅍ \pm n$ \\
\hline
\end{tabular}


ner, com $100 \mathrm{ml}$ de solução 0,03 normal em $\mathrm{PO}_{4}^{-3}$ (sendo 0,01 normal em $\mathrm{PO}_{4}^{-3}$ procedente do $\mathrm{H}_{3} \mathrm{PO}_{4}$ e 0,02 normal em $\mathrm{PO}_{4}^{-3}$, procedente do $\mathrm{KH}_{2} \mathrm{PO}_{4}$ ). Após a agitação, deixou-se em contato durante 24 horas e filtrou-se. Numa aliquota, determinou-se o $\mathrm{PO}_{4}^{-3}$, e por diferença da quantidade de $\mathrm{PO}_{4}{ }^{3-}$ determinada na prova em branco (numa alíquota da solução 0,03 normal em $\mathrm{PO}_{4}{ }^{3-}$, sem ter entrado em contato com o solo) calculou-se o número de equivalentes miligramas de $\mathrm{PO}_{4}^{-3}$ fixado.

$\mathrm{O}$ método analítico empregado foi o colorimétrico, baseado na formação do ácido molibdovanadofosfórico já discutido por diversos autores (KOENIG \& JOHNSON, 1942; KITSON \& MELLON, 1944 e BOLTZ \& LUECK, 1958).

Os reativos, soluções e padrões usados foram os seguintes:

Solução 0,03 normal em $\mathrm{PO}_{4}^{-3}$, sendo 0,01 normal em $\mathrm{H}_{3} \mathrm{PO}_{4}$ e 0,02 normal em $\mathrm{KH}_{2} \mathrm{PO}_{4}$.

Esta solução foi obtida a partir ide outras duas já preparadas. Uma delas era de normalidade conhecida em $\mathrm{H}_{3} \mathrm{PO}_{4}$, isto é, 0,150 $\mathrm{N}$ em $\mathrm{H}_{3} \mathrm{PO}_{4}$; a outra era 0,100 normal em $\mathrm{PO}_{4}^{-3}$, preparada, dissolvendo-se 4,5364 $\mathrm{g}$ de $\mathrm{KH}_{2} \mathrm{PO}_{4}$ sêco e completando-se o volume a 1 litro.

Solução padrão de $\mathrm{KH}_{2} \mathrm{PO}_{4}, 0,01$ normal em $\mathrm{PO}_{4}^{-3}$

Transferiu-se $50 \mathrm{ml}$ da solução 0,100 normal de $\mathrm{KH}_{2} \mathrm{PO}_{4}$ para um balão de $500 \mathrm{ml}$, foram adicionados $300 \mathrm{ml}$ de água destilada, $5 \mathrm{ml}$ de $\mathrm{H}_{2} \mathrm{SO}_{4}$, deixou-se esfriar e completou-se o volume.

Solução de molibdato de amônio a $10 \%$

Foram dissolvidos $100 \mathrm{~g}$ de sal em $600-700 \mathrm{ml}$ de água quente, transferiu-se para um balão de 1 litro, esperou-se esfriar e completou-se o volume.

Solução de metavanadato de amônio a 0,25\%

Foram dissolvidos $1,25 \mathrm{~g}$ de sal em $350-400 \mathrm{ml}$ de água em ebulição, transferiu-se para um balão de $500 \mathrm{ml}$, esperou-se esfriar e completou-se o volume.

Solução de $\mathrm{H}_{2} \mathrm{SO}_{4} 5 \mathrm{~N}$

Obtida a partir do $\mathrm{H}_{2} \mathrm{SO}_{4}$.

Relação entre a concentração em $P_{4}^{-8}$ e a densidade ótica das soluções

Foram medidos com microbureta 0,5, 1,0, 2,0, 3,0, 4,0, 5,0 e $6,0 \mathrm{ml}$ de solução padrão de fosfato, isto é, 0,010 normal em $\mathrm{PO}_{4}^{-3}$ e transferidos para balões volumétricos de $50 \mathrm{ml}$. Foram adicionados $30 \mathrm{ml}$ de água destilada, $5 \mathrm{ml}$ de solução de $\mathrm{H}_{2} \mathrm{SO}_{4} 5$ normal e o líquido foi homogeneizado. Em seguida, foram acrescentados $5 \mathrm{ml}$ da solução aquosa de metavanadato de amônio a $0,25 \%$, e 
mais $2 \mathrm{ml}$ de solução de molibdato de amônio a $10 \%$, homogeneizando-se após a adição de cada reativo. Completou-se o volume com água destilada, homogeneizou-se e depois de 10 minutos, determinou-se a transmissão porcentual, no aparêlho Spectronic 20, com comprimento de onda de $\mathbf{4 4 0}$ milimícrons. A transmissão foi convertida em densidade ótica e os valores obtidos foram relacionaidos com a concentração $\mathrm{PO}_{4}^{-3}$ dos padrões.

\section{3 - RESULTADOS OBTIDOS E DISCUSSÃO}

Os dados obtidos são apresentados no quadro 3 .

QUADRO 3 - Fixação de fósforo por diversas amostras de terra roxa e terra roxa misturada.

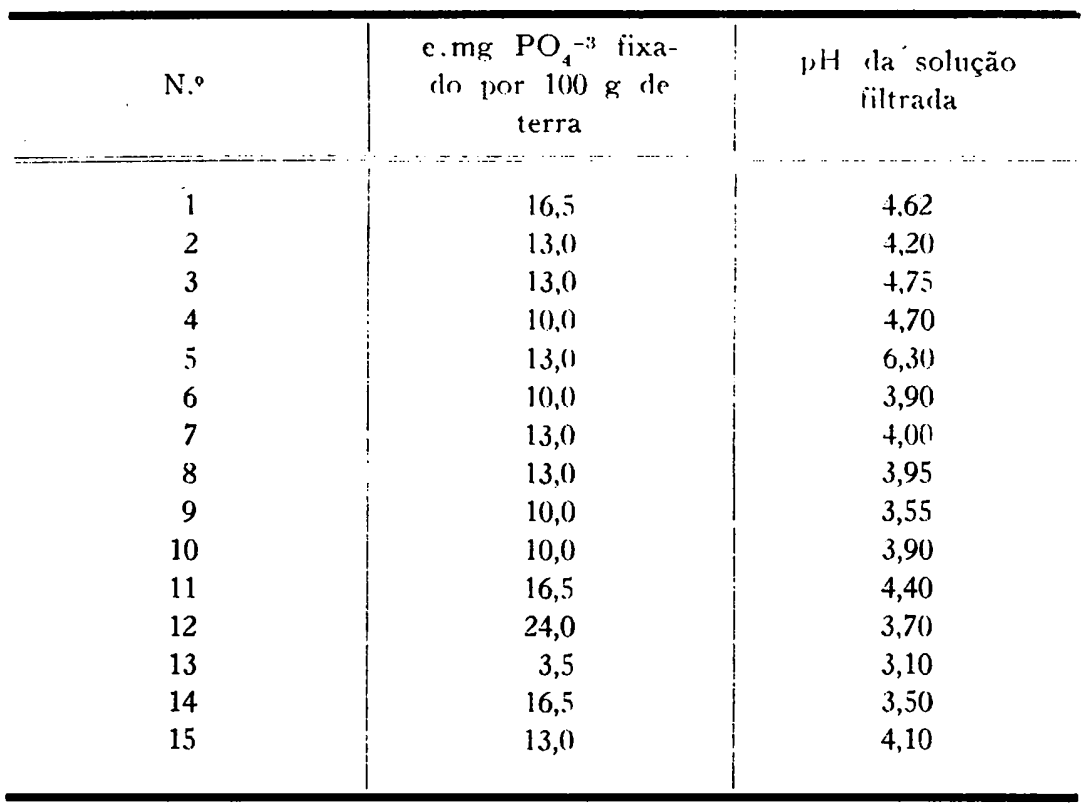

No quadro 3 estão apresentados os dados obtidos para a fixação do $\mathrm{PO}_{4}^{-3}$ em equivalentes-miligramas por $100 \mathrm{~g}$ de solo e o $\mathrm{pH}$ da solução depois de agitação e filtração. Pelos valores do $\mathrm{pH}$ da referida solução, pode-se concluir que a fixação foi promovida por fenômenos diversos da precipitação de fosfatos de cálcio ou de magnésio. Com certeza, o fenômeno predominante na fixação tenha sido o da adsorção dos ions fosfatos, promovida pelos óxidos hidratados de ferro e alumínio. 
Os valores obtidos para a fixação foram em geral, elevados e variaram de 3,5 a 24,0 equivalentes-miligramas de $\mathrm{PO}_{4}^{-3}$ por $100 \mathrm{~g}$ de terra. É interessante observar que o maior valor para a fixação tenha sido obtido pela terra $n .^{\circ} 12$, que apresenta o menor $\mathrm{pH}$, o menor indice de saturação em bases e a maior porcentagem de argila.

O menor valor para a fixação $\left(3,5 \mathrm{e} . \mathrm{mg}^{\circ} \mathrm{PO}_{4}^{-3}\right.$ por $100 \mathrm{~g}$ de terra) foi alcançado por uma terra muito ácida, com $\mathrm{pH}=4,70$, baixa saturação em bases $(17,3 \%)$, mas com uma baixa porcentagem de argila (17\%). Trata-se na realidade, de uma terra arenosa vermelha, porquanto a sua porcentagem de areia grossa e areia fina vai além de $80 \%$.

Em resumo, os dados obtidos são ainda pouco numerosos para se poder apresentar outras conclusões. Entretanto, já se pode formar uma idéia quantitativa da fixação de fosfato pela terra roxa e terra roxa misturada e, ainda mais, pode-se também admitir que o fenômeno de fixação não encontre explicação satisfatória na simples troca de ânions.

\section{4 - RESUMO E CONCLUSÕES}

A conversão das formas solúveis do fósforo em formas menos solúveis recebe a denominação de fixação. Muitos são os fatôres ou causas que afetam a fixação do fósforo pelo solo, como reações que originam compostos pouco solúveis (fosfatos de ferro, alumínio, cálcio, magnésio, manganês, etc.), adsorção do ion fosfato pelas partículas coloidais do solo, absorção do íon fosfato pelos vegetais inferiores e outros.

Todos os métodos de avaliação da capacidade de fixação do fósforo pelo solo são convencionais e fornecem resultados variáveis, de acôrdo com as condições de trabalho.

$\mathrm{O}$ método descrito e empregado é também convencional e resultou do estudo de uma série de fatôres, como a concentração da solução em fosfato e em ions hidrogênio, relação entre o pêso da amostra de terra e volume de solução, tempo de contato, etc.

A técnica adotada foi a seguinte:

$4 \mathrm{~g}$ de terra foram agitadas em balão de Erlenmeyer de $300 \mathrm{ml}$, durante 15 minutos a 30-40 rpm em aparelho, tipo Wagner, com $100 \mathrm{ml}$ de solução 0,03 normal em $\mathrm{PO}_{4}^{-3}$ (sendo 0,01 normal em $\mathrm{PO}_{4}^{-3}$ ) procedente do $\mathrm{H}_{3} \mathrm{PO}_{4}$ e 0,02 normal em $\mathrm{PO}_{4}^{-3}$ procedente do $\left.\mathrm{KH}_{2} \mathrm{PO}_{4}\right)$. Após a agitação, deixou-se em contato durante 24 horas e filtrou-se. Numa aliquota, determinou-se o $\mathrm{PO}_{4}^{-3}$ e por diferença da quantidade de $\mathrm{PO}_{4}^{-3}$ determinada na prova em branco (numa alíquota da solução 0,03 normal em $\mathrm{PO}_{4}^{-3}$, sem ter entrado 
em contato com o solo) calculou-se o número de equivalentes miligramas de $\mathrm{PO}_{4}^{-3}$ fixado.

O método analítico empregado foi o do ácido molibdovanadofosfórico e os dados obtidos em 15 amostras de terra, roxa e roxa misturada, permitem concluir que:

a) a capacidade de fixação de fósforo pela terra roxa e roxa misturada é relativamente elevada, tendo variado de 3,5 até 24,0 equivalente-miligramas de $\mathrm{PO}_{4}^{-3}$ por $100 \mathrm{~g}$ de terra. Das 15 amostras, 14 apresentaram uma fixação igual ou maior que 10 e. $\mathrm{mg}$ de $\mathrm{PO}_{4}^{-3}$ por $100 \mathrm{~g}$ de terra.

b) $\mathrm{o} \mathrm{pH}$ da solução 0,03 normal em $\mathrm{PO}_{4}{ }^{-3}$ procedente de $\mathrm{H}_{3} \mathrm{PO}_{4}$ e $\mathrm{KH}_{2} \mathrm{PO}_{4}$, depois de agitada com o solo e filtrada, evidencia que a fixação não é determinada por precipitação de fosfatos de cálcio e de magnésio. Outros fenômenos devem intervir e dentre êles, um dos principais deve ser o da adsorção de íons fosfatos pelos óxidos hidratados de ferro e alumínio.

\section{5 - SUMMARY}

Phosphate "fixation" is the convertion of soluble into insoluble phosphate in the soil.

There are many factors conditioning phosphate fixation by soil such as reactions originating less soluble compounds (phosphates of iron, aluminum, calcium, magnesium, etc.), $\mathrm{PO}_{4}^{-3}$ adsorption by the colloidal fraction of soils, $\mathrm{PO}_{4}^{-3}$ absorption by the soil microflora, etc.

Certain soils of the state of São Paulo (Brazil) are relatively rich in both iron and aluminum oxides. $\mathrm{PO}_{4}^{-3}$ fixation, using $\mathrm{P}_{31}$ and $P_{32}$ has been verified by researchers, specially with "Terra Roxa".

The known methods for fixation evaluation are conventional as this depends on phosphate solution concentration, $\mathrm{pH}$, time of contact between soil and solution, relation of sample weight to solution volume, shaking time, etc.

In this experiment, the following conventional method was used: $4 \mathrm{~g}$ of soil were shaken for 15 minutes at $30-40 \mathrm{rpm}$, in $300 \mathrm{ml}$ Erlenmeyer flask in a Wagner shaking machine, together with $100 \mathrm{ml}$ of 0,03 normal phosphate solution (being 0,01 normal as $\mathrm{PO}_{4}^{-3}$ contributed by $\mathrm{H}_{3} \mathrm{PO}$, and 0,02 normal as $\mathrm{PO}_{4}^{-3}$ from $\left.\mathrm{KH}_{2} \mathrm{PO}_{4}\right)$. After shaking it was set aside for 24 hours and then filtered. Phosphate was determined in a suitable aliquot of both the original solution (blank) and the soil extract, by the vanadomolibidic-phosphoric acid method. From phosphate concentra- 
tion in the blank minus phosphate concentration in the soil stract the rate of fixation by $100 \mathrm{~g}$ of soil was calculated.

The data obtained show that "Terra Roxa" and "Terra Roxa Misturada" have a fairly high $\mathrm{PO}_{4}^{-3}$ fixation capacity, varying from 10 to 24 milliequivalents of $\mathrm{PO}_{4}^{-3}$ per $100 \mathrm{~g}$ of soil.

\section{LITERATURA CITADA}

BOLTZ, D. F. \& C. H. LUECK, 1958 - Phosphorus. Em Colorimetric determination of nonmetals. Editado por D. F. Boltz. Interscience Publishers New York. pp. 29-46.

CATANI, R. A. e D. PELLEGRINO, 1957 - A fixação do fósforo em alguns solos do Estado de S. Paulo, estudada com o auxílio do fósforo radioativo $\mathbf{P}_{32}$. Rev. Agricultura, 32:237-252.

DEAN, L. A., 1949 - Fixation of soil phosphorus. En Advances in Agronomy vol. I - Editado por G. Norman. Academic Press. pp. 391-411.

HEMWALL, J. B. - 1957 - The fixation of phosphorus by soils. Em Advances in Agronomy, vol. IX. Editado por A. G. Norman. Acamic Press. pp. 95-112.

KITSON, R. E. \& M. G. MELLON, 1944 - Colorimetric determination of phosphorus as molybdivanadophosphoric acid. Ind. Eng. Chem. Anal. Ed. 16:379-383.

KOENIG, R. A. \& C. R. JOHNSON, 1942 - Colorimetric determination of phosphorus in biological materials. Ind. Eng. Chem. Anal. Ed. 14:155-156.

KURTZ, L. T., 1953 - Inorganic phosphorus in acid and neutral soils. Em Soil and Fertilizer phosphorus in crop nutrition. Editado por W. H. Pierce e A.. G. Norman. Academic Press, pp. 59-88.

OLSEN, S. R., 1953 - Inorganic phosphorus in alkaline and calcareous soils. Em Soil and Fertilizer phosphorus in crop nutrition. Editado por W. E. Pierre \& A. G. Norman. Academic Press, pp. 89-122. 
